\title{
Proposed Model for Performance Analysis of Fourth-generation Mobile Wireless Communication System
}

\author{
Weihua Li, ${ }^{1}$ Yang-Han Lee, ${ }^{2 *}$ Yi-Lun Chen, ${ }^{2}$ \\ Hsien-Wei Tseng, ${ }^{1}$ and Cheng-Fu Yang ${ }^{3,4^{* *}}$ \\ ${ }^{1}$ College of Mathematics and Information Engineering, Longyan University, Fujian 364012, China \\ ${ }^{2}$ Department of Electrical and Computer Engineering, Tamkang University, New Taipei City 251, Taiwan \\ ${ }^{3}$ Department of Chemical and Materials Engineering, National University of Kaohsiung, Kaohsiung 811, Taiwan \\ ${ }^{4}$ Department of Aeronautical Engineering, Chaoyang University of Technology, Taichung 413, Taiwan
}

(Received October 21, 2020; accepted February 3, 2021)

Keywords: IEEE $802.16 \mathrm{~m}$, long term evolution (LTE), channel model, orthogonal frequency division multiple access (OFDMA)

Both long term evolution (LTE) and IEEE $802.16 \mathrm{~m}$ technologies have the advantages of a high transmission rate and wide transmission bandwidth, and they play essential roles in modern mobile wireless communication systems. The information transmission processes between mobile users and base stations inevitably involve some propagation losses. The losses include shadow fading loss due to blocking by buildings and obstacles and the Doppler effect caused by the relative motion of base stations and mobile users, and these effects result in signal fading at the receiver terminals. To maintain good system performance and service quality, the selection of a suitable modulation format is necessary when wireless signals are transmitted. Different traffic services have their own requirements of service quality. On the basis of the specifications of the IEEE $802.16 \mathrm{~m}$ and LTE systems, we study their performance when the system parameters are varied. The system parameters include the system bandwidth, the transmitting powers of the base station and mobile, the antenna heights, the orthogonal frequency division multiplexing (OFDM) parameters, and the system load. We also analyze the system performances of the system bit error rate and block error rate (BER) due to the effects of path loss and noise.

\section{Introduction}

Wireless communication is a technology to deliver data from one point to another without using a signal or electrical wires, and it can transfer data using cellular, radio, satellite, and infrared. Today's common wireless networks originate from many models of wireless communication that evolved from radio and telegraph. ${ }^{(1,2)}$ A network service is an application running at the network layer and above, and it can provide functions including data storage, presentation, manipulation, and communication, which are implemented using a peer-topeer architecture or client server based on the communication layer of network protocols. Over time, people's requirements for network services have become multidimensional, for

\footnotetext{
*Corresponding author: e-mail: yhleepp@gmail.com

${ }^{* *}$ Corresponding author: e-mail: cfyang@nuk.edu.tw https://doi.org/10.18494/SAM.2021.3175
} 
example, improved service quality, and network services are becoming indispensable to our daily lives. As a result, enabling users to access network resources anytime and anywhere has become an important task of communication engineers. Earlier generations of wireless mobile communication can no longer meet current requirements. Meanwhile, network service providers would like to offer a more convenient wireless mobile communication system that can provide wider bandwidths in their signal transmission and expand the coverage area of their system. Both the IEEE $802.16 \mathrm{~m}$ and long term evolution (LTE) systems have their own interface standards, and both systems have the advantages of a high bandwidth, wide coverage area, and high transmission rate. Thus, for a newly proposed system, a task is to find the optimal system parameters, and then the communication service vendor can design new systems and investigate a system to meet the service requirements of users.

A fourth generation $(4 \mathrm{G})$ mobile wireless communication system is used to rapidly deploy networks consisting of a number of sensor nodes. Wireless sensor networks, cellular systems, and local/personal area networks are used to support localization functionality. The IEEE 802.16m and LTE systems were proposed on the basis of the Third Generation Partnership Project (3GPP) and 802.16 systems, and we study their performances when different numbers of users use them simultaneously. In this paper, we study the performances of the IEEE $802.16 \mathrm{~m}$ and LTE systems for a $4 \mathrm{G}$ wireless communication system when their parameters are varied. The novelty of this paper is that we use the 3GPP and LTE systems to construct a traffic model for simulation that is close to the actual network usage. A traffic model is investigated to present the traffic flows of an actual wireless mobile communication system. Then we determine the quality of services, which is used to judge whether a base station can provide adequate services. In this study, the services of the considered traffic flows include common network services such as Voice over Internet Protocol (VoIP), video stream, HyperText Transfer Protocol (HTTP), and File Transfer Protocol (FTP), and each system has its own requirement of service quality. In this study, we first consider the problems of fading and path loss between different base stations and mobile users to reflect a real communication environment. Then, we study the system capacity by selecting a suitable modulation format for the performance simulation, which is applied to the performance system through an adaptive modulation and coding (AMC) scheme.

\section{System Parameters}

\subsection{IEEE $802.16 \mathrm{~m}^{(3-8)}$}

In time division duplex situation of the IEEE $802.16 \mathrm{~m}$ system, the superframe is $20 \mathrm{~ms}$ and each superframe consists of four frames with a duration of $5 \mathrm{~ms}$. The first frame has a superframe header; each frame is then divided into uplink (UL) and downlink (DL) subframes with a ratio of 5:3. There are three possible types of subframes: type 1 consists of six orthogonal frequency division multiplexing (OFDM) symbols, and types 2 and 3 have seven and three symbols, respectively, and the different types have different sizes of the cyclic prefix (CP) to form various types of subframes. Table 1 lists the system parameters of IEEE $802.16 \mathrm{~m}$. 
Table 1

IEEE $802.16 \mathrm{~m}$ parameters.

\begin{tabular}{|c|c|c|c|c|c|c|c|}
\hline \multicolumn{3}{|c|}{ Nominal channel bandwidth (MHz) } & 20 & 10 & 8.75 & 7 & 5 \\
\hline \multicolumn{3}{|l|}{ Oversampling factor } & $28 / 25$ & $28 / 25$ & $8 / 7$ & $8 / 7$ & $28 / 25$ \\
\hline \multicolumn{3}{|c|}{ Sampling frequency $(\mathrm{MHz})$} & 22.4 & 11.2 & 10 & 8 & 5.6 \\
\hline \multicolumn{3}{|l|}{ FFT size } & 2048 & 1024 & 1024 & 1024 & 512 \\
\hline \multicolumn{3}{|c|}{ Sub-carrier spacing $(\mathrm{kHz})$} & 10.9375 & 10.9375 & 9.765625 & 7.8125 & 10.9375 \\
\hline \multicolumn{3}{|c|}{ Useful symbol time $T u(\mu \mathrm{s})$} & 91.429 & 91.429 & 102.4 & 128 & 91.429 \\
\hline \multirow{3}{*}{$\begin{array}{l}\text { Cyclic prefix }(\mathrm{CP}) \\
T g=1 / 8 T u\end{array}$} & \multicolumn{2}{|c|}{ Symbol time $T s(\mu \mathrm{s})$} & 102.857 & 102.857 & 115.2 & 144 & 102.857 \\
\hline & \multirow[t]{2}{*}{ TDD } & $\begin{array}{l}\text { Number of OFDM } \\
\text { symbols per frame }\end{array}$ & 47 & 47 & 42 & 33 & 47 \\
\hline & & $\mathrm{TTG}+\mathrm{RTG}(\mu \mathrm{s})$ & 165.714 & 165.714 & 161.6 & 248 & 165.714 \\
\hline \multirow{3}{*}{$\begin{array}{l}\text { Cyclic Prefix (CP) } \\
T g=1 / 8 T u\end{array}$} & \multicolumn{2}{|c|}{ Symbol time $T s(\mu \mathrm{s})$} & 97.143 & 97.143 & 108.8 & 1.36 & 97.143 \\
\hline & \multirow[t]{2}{*}{ TDD } & $\begin{array}{l}\text { Number of OFDM } \\
\text { symbols per frame }\end{array}$ & 50 & 50 & 44 & 35 & 50 \\
\hline & & $\mathrm{TTG}+\mathrm{RTG}(\mu \mathrm{s})$ & 142.853 & 142.853 & 212.8 & 249 & 142.853 \\
\hline
\end{tabular}

\subsection{LTE system}

LTE has been developed to operate on paired spectra for frequency duplex division (FDD). ${ }^{(8-13)}$ The FDD format of LTE technology has ten $1 \mathrm{~ms}$ subframes in a $10 \mathrm{~ms}$ frame, with each subframe consisting of two time slots, and each time slot can be classified into a short CP or long $\mathrm{CP}$ according to its length. There are six OFDM symbols in a short $\mathrm{CP}$ frame and seven OFDM symbols in a long CP frame. The system parameters of LTE are shown in Table 2.

\subsection{Traffic model}

Four traffic services are considered in our system, and with the four traffic services, we set up a simulation environment that is similar to the actual network condition. The traffic capacity of a base station in one superframe is based on the service quality, service priority, and the probability of demand for each service in the network. A real-time service has the highest service priority, followed by VoIP, video stream, HTTP, and FTP in decreasing order of priority. Video stream is a bidirectional service and its capacity is limited by the uplink bandwidth. In this simulation, we assume that each user can request any of the four available service systems, and the probability that each service system will be requested is listed in Table 3 . When the capacity in a frame cannot meet the demand for all services, then some services need to be postponed until the next frame that can provide the services. If the delay time of the demanded services is over the threshold limitation, then the packet can be determined as lost.

\section{Channel Model}

\subsection{Channel model for wireless communication}

When signals are transmitted through the communication channels, they are faded by many factors, for example, when signals are reflected, multipath effects occur. The signals can be 
Table 2

LTE system parameters.

\begin{tabular}{lccccccc}
\hline Transmission BW $(\mathrm{MHz})$ & 1.25 & 2.5 & 5 & 10 & 15 & 20 \\
\hline Sub-frame duration & \multicolumn{7}{c}{$0.5 \mathrm{~ms}$} \\
\hline Sub-frame spacing $(\mathrm{MHz})$ & \multicolumn{7}{c}{15} \\
\hline Sampling frequency $(\mathrm{MHz})$ & 1.92 & 3.84 & 7.68 & 15.36 & 23.04 & 30.72 \\
\hline FFT size & 128 & 256 & 512 & 1024 & 1536 & 2048 \\
\hline \multicolumn{7}{l}{ OFDM symbols per slot (short/long CP) } \\
\hline \multirow{2}{*}{ CP length $(\mu$ s/sample) } & \multirow{2}{*}{ Short } & $(4.69) \times 6$ & $(4.69) \times 6$ & $(4.69) \times 6$ & $(4.69) \times 6$ & $(4.69) \times 6$ & $(4.69) \times 6$ \\
\cline { 2 - 9 } & Long & $(5.21) \times 1$ & $(5.21) \times 1$ & $(5.21) \times 1$ & $(5.21) \times 1$ & $(5.21) \times 1$ & $(5.21) \times 1$ \\
\hline
\end{tabular}

Table 3

Mixed probabilities of traffic.

\begin{tabular}{lcccc}
\hline & VoIP & FTP & HTTP & NRTV \\
\hline NGMN Traffic Mix & $35 \%$ & $15 \%$ & $25 \%$ & $25 \%$
\end{tabular}

NGMN: next-generation mobile networks, NRTV: near real-time video.

diffracted and scattered by buildings, forests, and the terrain, then the received signals are faded and cannot be correctly detected. ${ }^{(14-18)}$ The Doppler effect occurs when there is relative motion between the mobile station and the base station, which causes frequency modulation in the received signals, increasing the uncertainty in the quality of the received signals. Wireless communication channels are usually analyzed and simulated from channel statistical characteristics, and they have been discussed in many studies on the analysis of wireless communication systems. The fast fading model and path loss model are discussed in this paper and applied to the development of the proposed software, then we create a simulation of a situation that is close to the actual communication environment. The received power of mobile users or base stations can be expressed as

$$
P(\mathrm{dBm})=P_{t}+G_{t}+G_{r}-P L+F
$$

$P_{t}: \quad B S / M S$ transmission power

$G_{t}: B S / M S$ transmission antenna gain

$G_{r}: B S / M S$ receiver antenna gain

$P L$ : Path loss

$F$ : Fast fading

where $B S$ is the base station and $M S$ is the mobile station. The COST-231 path loss model and Rayleigh fading model have been used in calculating the received signal strength and are discussed in the following.

\subsubsection{COST-231 model}

When signals are generated and transmitted, the signal strength has different losses, which are dependent on the communication environment and transmission distance, and the received signal strength is a random variable. The COST-231 Hata model is a radio propagation model 
that extends the urban Hata model and it can cover a larger range of frequencies of up to $2 \mathrm{GHz}$. The COST-231 Hata model is usually used to describe the average received signal strength. ${ }^{(13)}$ The COST-231 Hata model in Eq. (2) is an empirical formula compiled and developed from many measurements in field trials and can be principally applied in urban areas and for carrier frequencies in the range of 150 to $1500 \mathrm{MHz}$.

$$
\begin{aligned}
P L(\mathrm{~dB})= & \left(44.9-6.55 \log _{10}\left(h_{b s}\right)\right) \log _{10}\left(\frac{d}{1000}\right)+45.5+\left(35.46-1.1 h_{m s}\right) \log _{10}(f) \\
& -13.82 \log _{10}\left(h_{b s}\right)+0.7 h_{m s}+C
\end{aligned}
$$

$f: \quad$ Carrier frequency $(\mathrm{MHz})$

$d$ : Distance in meters between the base station and the mobile user

$C$ : Constant depending on the communication environment, which is $3 \mathrm{~dB}$ for an urban environment

$h_{B S}$ : Base station effective antenna height

$h_{M S}:$ Mobile user effective antenna height

IEEE $802.16 \mathrm{~m}$ and LTE bands cannot directly use the frequency band considered in Eq. (2) for their operating frequencies, and Eq. (2) must be modified to Eqs. (3) and (4) for urban and suburban areas, respectively, where $f$ is the frequency in GHz. These equations were proposed by Jakes to extend the path loss model to the frequency band of $2-6 \mathrm{GHz}^{(17)}$

$$
\begin{gathered}
P L_{\text {urban }}(\mathrm{dB})=\left(44.9-6.55 \log _{10}\left(h_{b s}\right)\right) \log _{10}(d)+26.46+5.83 \log _{10}\left(h_{b s}\right)+26 \log _{10}(f / 2) \\
P L_{\text {suburban }}(\mathrm{dB})=P L_{\text {urban }}-2\left[1.5528+\log _{10}(f)\right]^{2}-5.4
\end{gathered}
$$

\subsubsection{Fast fading}

When a signal is transmitted, it will be affected by the communication environment, such as by diffraction, refraction, scattering, and the relative velocity of the motion between the base station and the mobile receiver. The two factors with the greatest effect on fast fading are the multipath effect and the Doppler effect, which make the received signal strength change with time and generate signal dispersion and frequency modulation in the received signal.

\subsection{Analysis of signal-to-noise ratio (SNR) versus distance}

As the received SNR for the maximum possible transmission distance between the transmitter and receiver is considered, it can be used to determine suitable modulation and coding rates. The maximum possible strength of the received signal can be determined from the $S N R$ at the receiver terminal, while the noise power $N$ can be determined as

$$
N=B W \frac{4.0 p W}{\mathrm{GHz}},
$$


where $B W$ is the system bandwidth. From the path loss model shown in Eqs. (3) and (4), we find the fast fading model from the received signal strength of the base station or mobile user, and then the $S N R$ at the receiver terminal can then be calculated as

$$
S N R(\mathrm{~dB})=P(\mathrm{dBm})-N(\mathrm{dBm}),
$$

where $P$ is the $B S / M S$ receiver power at the base station or mobile user and $N$ is the noise power.

\section{Software Simulation}

\subsection{Software simulation for interface}

The simulation of base station performance is executed using a graphical user interface (GUI). First, we need to generate the traffic service demand of each mobile user. Next, the arrangements of services are dependent on the scheduler at the base stations, and signals are transmitted from suitably selected modulation and coding schemes. Then, the block error probability and average traffic service quality are calculated, and if the information needs to be retransmitted, the most suitable retransmission mechanism is selected. After all packets are transmitted, the final system transmission rates are also calculated. As shown in Fig. 1, the interface consists of six parts, each part having its own parameters and functions. The interface of each part and its associated parameters are described in the following:

(a) Path loss mode: The simulation of the path loss consists of two communication environments: urban and suburban.

(b) Selection of base station parameters: Three subsystem parameters are considered: OFDM parameters, mobile equipment parameters, and base station parameters.

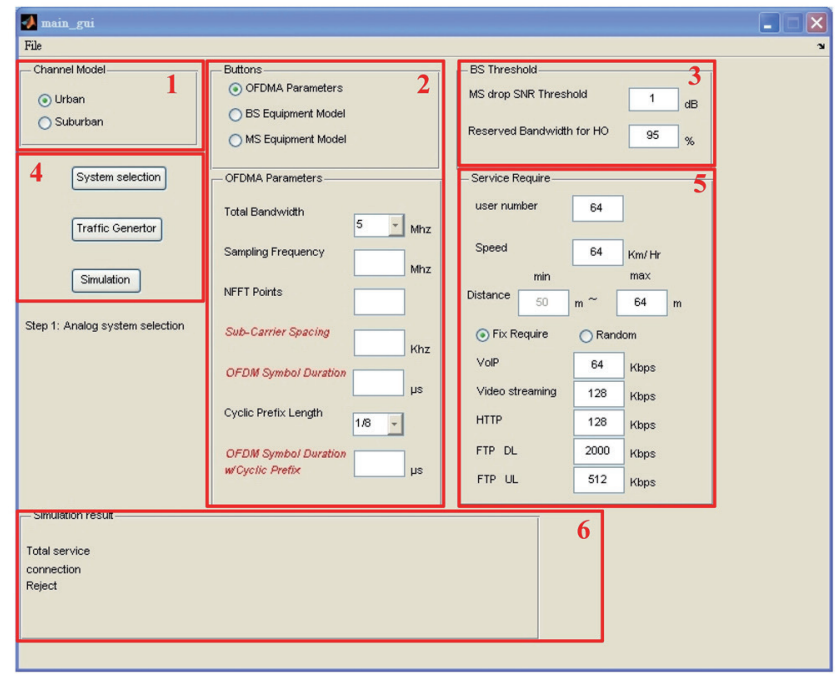

Fig. 1. (Color online) Main interface. 
(c) Limitations in the selection of system parameters: The following parameters have certain restrictions in their utilization in the system simulation, such as the authorization of the users to enter into the system, the bandwidth allocated for handoff (the handoff bandwidth threshold), and the mobile station drop threshold (base station downlink SNR threshold)

(d) System operations: They consist of three procedures in the running of this software. First, the simulation system is generated, then information relating to traffic flows is generated and the simulation proceeds, and finally the simulation results are outputted into the documents.

(e) Parameter restrictions in the traffic services: This process sets up the system parameters. This system allows the simulation operator to set the number of users allowed to enter the system, the user requirements for various services, the mobile moving velocity, and the transmission distance between the base stations and the mobile users.

(f) Outputs of the simulation results: In this process, the system displays the total service traffic and the amount of services permitted and restricted in the base stations.

Figure 2 shows the interface of the service and capacity counting tool, which is divided into seven parts, with each part listed below:

(a) System information: This part sets up the basic input parameters of the system information, including the frame length, allowed bandwidth, fast Fourier transform sampling number, downstream usage ratio, and transmission modulation, and the output information is the useful transmission bandwidth.

(b) Service requirements: The parameters in this part belong to the demand setting, which can set up different services by users, including the number of users, simulation time, and the demand for the different services.

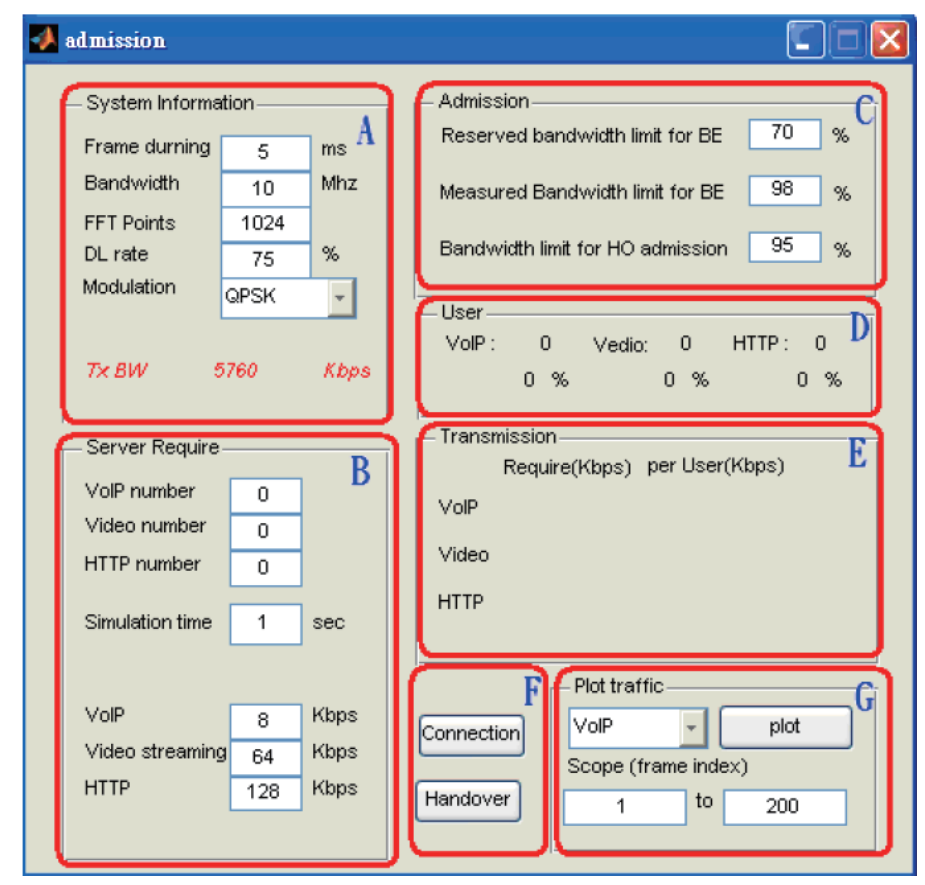

Fig. 2. (Color online) Interface of service and capacity counting tool. 
(c) Access parameters: There are three parameters associated with access permission of the users, including the access to the bandwidth of changing users, the access to the bandwidth of the reserved BE services, and the access to the bandwidth of the measured BE services.

(d) User information: This corresponds to the output parameters and displays the numbers of users accessing services and their relative bandwidths.

(e) Transmission information: This also corresponds to the output parameters. In the simulation time, this displays the randomly generated total demand and the average demand of each user calculated in the model.

(f) User login method: Two buttons are used to represent the different models for user login, i.e., general login and login by changing the user, and the two models have different admission criteria of access authority.

(g) Plotting parameters: These parameters are used to set up the demand of the output flow rate, and the drawing range and the class of services can also be chosen.

\subsection{Selection of parameters for base stations}

The parameters used in orthogonal frequency division multiple access (OFDMA) are total bandwidth (TBW), sampling frequency (SF), NFFT points (NFTT), sub-carrier spacing (SCs), OFDM symbol duration (SD), cyclic prefix length (CPL), and OFDM symbol duration w/cyclic prefix (SDwcp). The parameters considered in the BS equipment model are operating frequency $(\mathrm{F})$, channel bandwidth (BW), BS transmission power, BS antenna gain, BS height, and superframe/ frame duration. The parameters considered in the MS equipment model are MS transmission power, MS antenna gain, and MS height.

\subsection{Simulation flows}

Figure 3 is a flow chart of the proposed simulation, which is divided into three processes. First, the system, i.e., IEEE $802.16 \mathrm{~m}$ or LTE, must be selected because the different systems have different parameters, which will affect the selected parameters in the second process. The four different traffic services described above are then selected, followed by the total number of users and mobile velocity. After these parameters are selected and the traffic generator button is pushed, the traffic models are generated for a total of $N$ users. In the third process, the

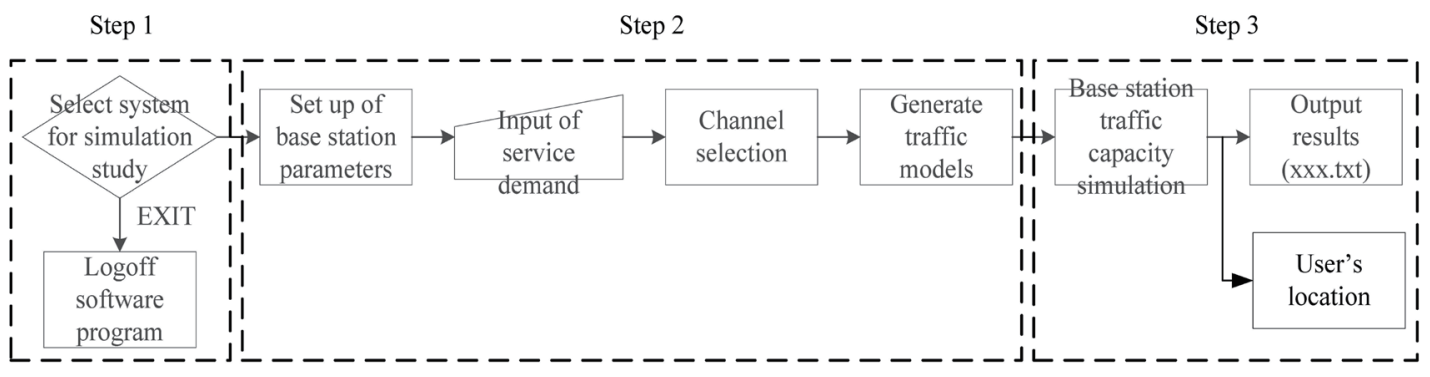

Fig. 3. Simulation flow chart. 
base stations with the total traffic are simulated, which are based on the transmission distance between the mobile users and the base stations, the path loss, and the MediaTek cloud sandbox (MCS) scheme of each user's transmission, then the system block error rate (BER) is calculated. The outputs display each user's modulation format, location, SNR value, etc.

The simulation parameters of the investigated system are listed in Table 4. Each user can have at most four different types of service. As shown by the output results in Fig. 4, the service traffic capacity has a normal distribution. A schematic diagram of locations of the users is shown in Fig. 5. The system specification agencies of IEEE 802.16 and 3GPP have both proposed standards for wireless network interfaces, and both of them have the advantages of a high transmission speed, high bandwidth, and large coverage. The proposed system is constructed on the basis of the IEEE $802.16 \mathrm{~m}$ and LTE systems, and we simulate and analyze

Table 4

Simulation parameters.

\begin{tabular}{lc}
\hline Parameter & Value \\
\hline Sampling frequency & $5.6 \mathrm{MHz}$ \\
NFFT points & 512 \\
GI & $1 / 8$ \\
MIMO & $2 \mathrm{X} 2$ \\
Channel estimation & $\mathrm{MMSE}$ \\
Number of users & 30 \\
\hline
\end{tabular}

\begin{tabular}{|c|c|c|c|c|c|c|}
\hline Connection State & Server & Requirement(kbps) & Distance(M) & $\operatorname{SNR}(\mathrm{dB})$ & Modulation & Code Rate \\
\hline User Number: 1 & Speed: $55 \mathrm{~km} / \mathrm{h}$ & & & & & \\
\hline RU Threshold & HTTP & $128 / 0$ & 386 & $10.52 / 3.52$ & $4 / 0$ & $0.500 / 0.000$ \\
\hline OK & VoIP & $64 / 64$ & 386 & $10.52 / 3.52$ & $4 / 2$ & $0.500 / 0.500$ \\
\hline RU Threshold & DL FTP & $2000 / 0$ & 386 & $10.52 / 3.52$ & $4 / 0$ & $0.500 / 0.000$ \\
\hline $\mathrm{OK}$ & UL FTP & $0 / 512$ & 386 & $10.52 / 3.52$ & $0 / 2$ & $0.000 / 0.500$ \\
\hline User Number: 2 & Speed: $44 \mathrm{~km} / \mathrm{h}$ & & & & & \\
\hline RU Threshold & HTTP & $128 / 0$ & 310 & $13.95 / 6.95$ & $4 / 0$ & $0.750 / 0.000$ \\
\hline User Number: 3 & Speed: $4 \mathrm{~km} / \mathrm{h}$ & & & & & \\
\hline OK & VoIP & $64 / 64$ & 305 & $14.52 / 7.52$ & $4 / 2$ & $0.750 / 0.750$ \\
\hline RU Threshold & HTTP & $128 / 0$ & 305 & $14.52 / 7.52$ & $4 / 0$ & $0.750 / 0.000$ \\
\hline OK & Vedio & $128 / 0$ & 305 & $14.52 / 7.52$ & $4 / 0$ & $0.750 / 0.000$ \\
\hline $\mathrm{OK}$ & UL FTP & $0 / 512$ & 305 & $14.52 / 7.52$ & $0 / 2$ & $0.000 / 0.750$ \\
\hline User Number: 4 & Speed: $15 \mathrm{~km} / \mathrm{h}$ & & & & & \\
\hline OK & DL FTP & $2000 / 0$ & 190 & $21.64 / 14.64$ & $6 / 0$ & $0.750 / 0.000$ \\
\hline OK & Vedio & $128 / 0$ & 190 & $21.64 / 14.64$ & $6 / 0$ & $0.750 / 0.000$ \\
\hline $\mathrm{OK}$ & Vedio & $128 / 0$ & 190 & $21.64 / 14.64$ & $6 / 0$ & $0.750 / 0.000$ \\
\hline User Number: 5 & Speed: $37 \mathrm{~km} / \mathrm{h}$ & & & & & \\
\hline RU Threshold & HTTP & $128 / 0$ & 467 & $7.71 / 0.71$ & $2 / 0$ & $0.750 / 0.000$ \\
\hline User Number: 6 & Speed: $33 \mathrm{~km} / \mathrm{h}$ & & & & & \\
\hline OK & VoIP & $64 / 64$ & 157 & $24.64 / 17.64$ & $6 / 4$ & $0.750 / 0.750$ \\
\hline OK & Vedio & $128 / 0$ & 157 & $24.64 / 17.64$ & $6 / 0$ & $0.750 / 0.000$ \\
\hline $\mathrm{OK}$ & Vedio & $128 / 0$ & 157 & $24.64 / 17.64$ & $6 / 0$ & $0.750 / 0.000$ \\
\hline
\end{tabular}

Fig. 4. Output report. 


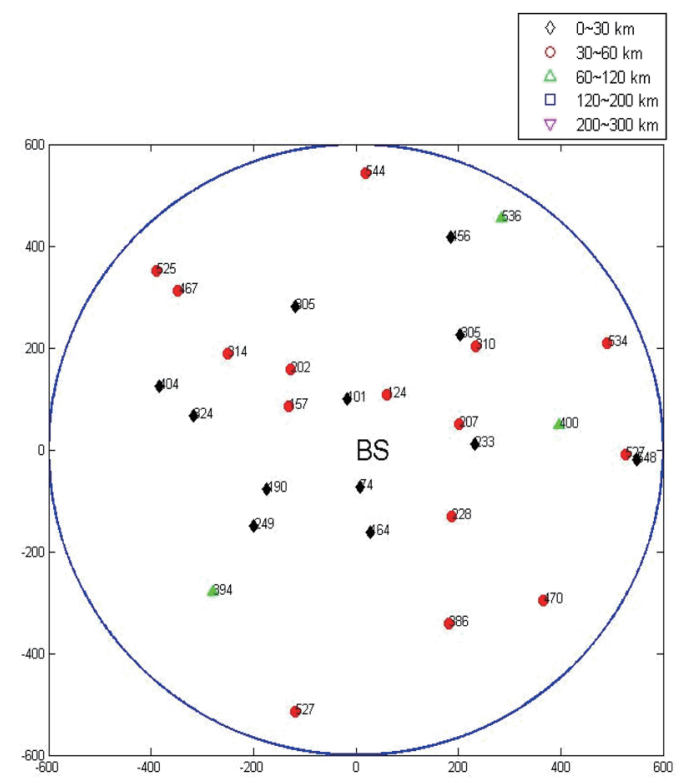

Fig. 5. (Color online) Schematic diagram of locations.

the performances of the base station using the frame time, bandwidth, and SF. Each service has its own quality of service, for which we consider the fading and path loss between each mobile station and base station. Then we can choose the most appropriate transmission mode for simulation via the AMC mechanism to obtain the usage efficiency of the base station that is closest to its maximum capacity. Then we use the GUI as the software development platform of the proposed simulation process. We use the variable SNR channel to decide the modulation encoding rate and the AMC mechanism to decide the modulation and coding of each user. The working principle is that we choose the system with the higher transmission rate to transmit data, and then each user feeds back the SNR signals to the base station once the user has received the message from the base station. When the base station receives the feedback SNR value, it chooses the most suitable modulation and coding via the mechanism of $\mathrm{AMC}$, and then the transmission error rate is reduced effectively. The proposed software can simulate the case that when a base station has different parameters, qualifications, and channel interference, it can still guarantee the quality of the maximum possible number of services. By examining the effect of the channel on signal attenuation, we find that when multiple users are using the network, their signal can find a suitable MCS to improve the performance of the base station.

\section{Conclusion}

In this paper, a simulation of base station traffic capacity has been developed to evaluate the maximum service capacity that a base station can provide under various conditions, such as different sets of system parameters, communication environments, and classes of channel fading. These simulation results were obtained under the restrictions that a service quality of a certain level must be guaranteed with limitations in the values of some system parameters. 
We found through the study of the fading characteristics of signals that when the signals were passed and transmitted through the channel with many users in the system, the system performance could be optimized or improved when a suitable MCS mechanism was selected for each user. The proposed software showed that we can simulate a base station with different parameters, qualifications, and channel interference while still guaranteeing the quality of the maximum limited number of services. Using the proposed simulation, we hope to provide valuable and effective recommendations for the choice of system parameters for service providers in the early setup of their system and to help them complete the system installation in the shortest possible time period.

\section{Acknowledgments}

This study was supported by Longyan University's Qi Mai Science and Technology Innovation Fund Project of Longyan City (2017QM0201 and 2018LYQM0202); Longyan University's Qi Mai Science and Technology Innovation Fund Project of Liancheng (2018)132 and Shanghang (2019SHQM05) County, Longyan; and Longyan University's Research and Development Team Fund (2018)8, Great Project of Production, Teaching, and Research of Fujian Provincial Science and Technology Department (2019H6023). This study was also partially supported by the National Science Council, Taiwan, under contracts NSC 99-2219-E-009-014, NSC 97-2221-E-032-027-MY3, MOST 109-2221-E-390-023, MOST 109-2221-E-390-023, and MOST 109-2321-B-001-013, and the Ministry of Education, Taiwan, project of "Agriculture Artificial Intelligence Internet of Things (AgAIoT)".

\section{References}

1 T. K. Sarkar, R. Mailloux, A. A. Oliner, M. Salazar-Palma, and D. L. Sengupta: History of Wireless (Wiley-IEEE Press, 2006).

2 J. Wheat, R. Hiser, J. Tucker, A. Neely, and A. McCullough: Designing A Wireless Network (Elsevier Inc. Amsterdam, Netherlands, 2001).

3 Y. C. Chan and S. H. Hsu: IET Int. Conf. Wireless Sensor Network 2010 (IET-WSN, 2010) 249.

4 W. Chen and J. Lin: IEEE Trans. Vehicular Technol. 63 (2014) 2208.

5 C. Kao, S. Yang, and H. Chen: IEEE Trans. Mobile Compu. 13 (2014) 2870.

6 K. Chu, T. Huang, J. Jhou, and S. Cheng: 2013 3rd Int. Conf. Consumer Electronics, Communications and Networks (2013) 542-545.

7 IEEE Standard for Local and metropolitan area networks Part 16: IEEE Std 802.16m-2011 (Amendment to IEEE Std 802.16-2009) 1-1112.

8 H. W. Tseng, Y. H. Lee, C. Y. Lo, and M. H. Lee: 2015 Int. Conf. Consumer Electronics-Taiwan (ICCE, 2015) 436.

9 F. Gao, Y. Wang, and Y. Zhang: 2017 IEEE Int. Conf. Signal Processing, Communications and Computing (ICSPCC, 2017) 1-4.

10 J. H. Choi, H. K. Chung, H. Lee, J. Cha, and H. Lee: 2006 IEEE Vehicular Technology Conf. (2006) 1.

11 W. Zhang, J. Chen, Y. Kuo, and Y. Zhou: IEEE Trans. Vehicular Technol. 68 (2019) 9747.

12 G. Zhao and L. Xing: 2017 Annu. Reliability and Maintainability Symp. (RAMS, 2017) 1.

13 F. A. P. de Figueiredo, F. Mathilde, L. R. Pizzini, F. Figueiredo, D. Carrillo, and I. Moerman: 2017 IEEE XXIV Int. Conf. Electronics, Electrical Engineering and Computing (INTERCON, 2017) 1.

14 E. Damosso and L. M. Correia: Cost Action Final Report (1999).

15 M. Gudmundson: Electron. Lett. 27 (1991) 2145.

16 R. Prakash and V. V. Venugopal: IEEE J. Selected Areas Commun. 18 (2000) 2456.

17 W. C. Jakes: Microwave Mobile Communication (IEEE Press, New York, 1974).

18 P. S. Rha: Electron. Lett. 34 (1998) 237. 